The errors at the 27 arguments corresponding to those listed under Legendre $8(\alpha, \gamma)$ are given at the bottom of p. 280, the last digit being always in the fourteenth place. The list probably omits no error of more than ten final units in Verhulst. The corrections are slightly provisional, but are liable to errors of only a unit or so in the last place, as I later applied a few small corrections to log $K$ not listed under LEGENDRE $8(\alpha)$, and kept extra decimals in the calculations when I knew them. At $1^{\circ} .1$ LEGENDRE's errors in $\log K$ and $\log$ $K^{\prime}$ nearly cancel, so that Verhulst is nearly correct.

( $\beta) \log \log (1 / q), \theta=\left[15^{\circ}\left(0^{\circ} .1\right) 45^{\circ} ; 12 \mathrm{D}\right]$.

There are exactly four gross errors:

\begin{tabular}{crrr}
$\theta$ & \multicolumn{1}{c}{$D$} & For & Read \\
$15^{\circ} .5$ & 11 & 4 & 3 \\
21.0 & $9-10$ & 22 & 16 \\
30.9 & 8 & 6 & 5 \\
37.7 & 12 & 9 & 0
\end{tabular}

The correction to the last figure at $37^{\circ} .7$ is approximate, being that which brings Verhulst's value into agreement with LEGENDRE's $\log K$ and $\log K^{\prime}$, and also rectifies the differences. When the above corrections are made, the table differences very well, especially when it is remembered that it was formed by subtracting Legendre's log $K$ from his $\log K^{\prime}$ (and adding a constant, which, however, will not affect the differences); even if LEGENDRE had no rounding errors, Verhulst's values could frequently be wrong by one final unit. A similar remark applies to the $14 \mathrm{D}$ portion considered in $(\alpha)$ above. Verhulst's values are unaffected by the two gross errors (one obvious) at $24^{\circ} .9$ and $30^{\circ} .9$ in LEGENDRE 5 , listed under LEGENDRE $8(\beta)$, his error at $30^{\circ} .9$ being quite different; thus he probably used LEGENDRE 3 .

WAYNE 1.

(a) $E, k^{\prime}=[0(.01) 1 ; 3 \mathrm{D}]$.

Comparison with Schlömilch 1 and Schmidt 1 shows two slight rounding errors; at $k^{\prime}=.20$, for 1.050 , read 1.051 , and at $k^{\prime}=.81$, for 1.426 , read 1.425 .

\title{
The Memory Tube and Its Application to Electronic Computation
}

In a paper presented on 10 June 1947 at the IRE Electron Tube Conference, the author described a novel electronic device which is called the Memory Tube. ${ }^{1}$ The Memory Tube has the ability to record electrical signals in the form of a charge pattern deposited by an electron beam on a dielectric target, to store this information for any desired period of time, and to reproduce the stored information in the form of electrical signals as many times as desired, without erasing the recorded information. The recorded charge pattern or any part of it can be erased whenever desired. These characteristics of the Memory Tube make it useful for many applications, including electronic computation. The purpose of this paper is to review the basic principles of operation of the Memory Tube and to describe a special form suitable for use as a memory device in an electronic computer.

The Memory Tube resembles a conventional cathode-ray tube. At one end of an elongated envelope are placed one or more electron guns. At the other end is placed a dielectric target, which ordinarily consists of a glass plate coated with a suitable material, such as phosphor. In front of the target a fine-mesh metal screen is placed at a distance of a few thousandths of an inch from the target. The screen serves to control the electric field at the 
target surface and to collect secondary electrons emitted from the target. The charge pattern is deposited by a well-focused electron beam which "writes" on the particular target element to which it is directed. An auxiliary electron gun producing a diffuse electron stream continuously bombards the whole area of the target. The velocity of electrons in this "holding" beam is so adjusted that electrons arriving at positive areas tend to maintain those areas at collector potential, while electrons arriving at negative areas drive these areas to holding gun-cathode potential. This result is achieved because of the secondary emission characteristics of the target,-
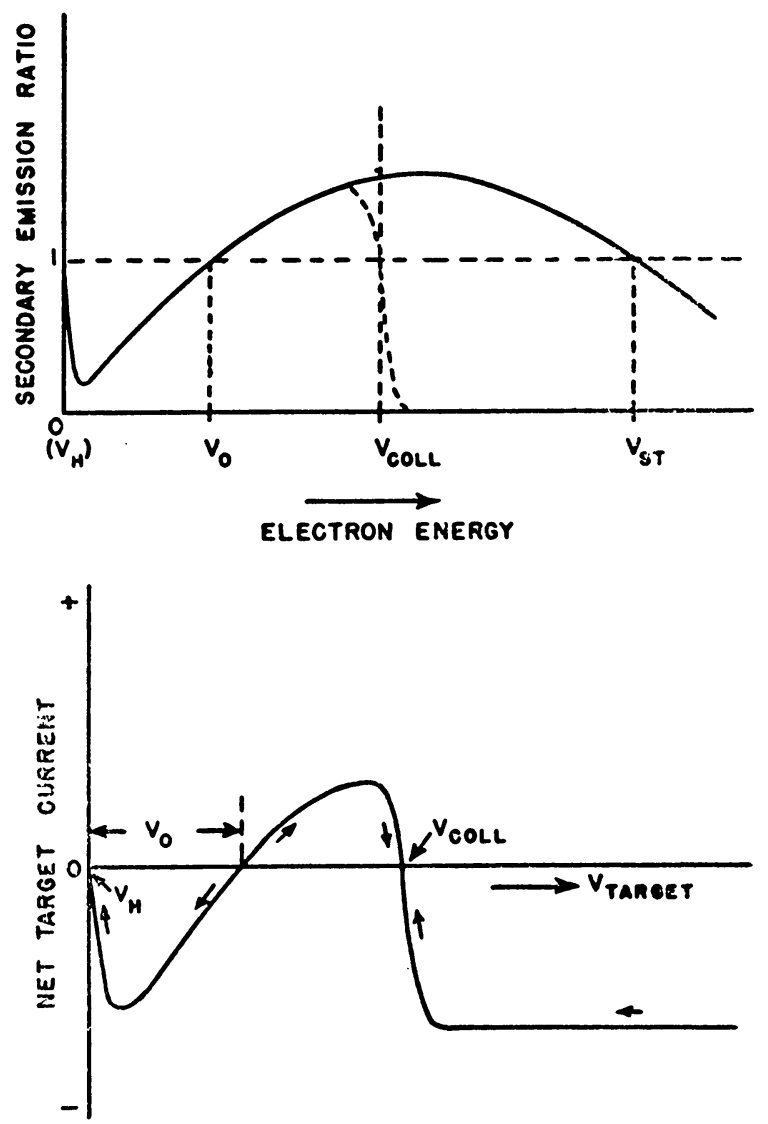

Fig. 1. Secondary Emission Characteristics of the Memory Tube Target.

namely, that the net current flowing to the target element is negative when the electron velocity is below a critical value $V_{0}$ corresponding to unity ratio of secondary emission, and is positive when the velocity exceeds this critical value (see Fig. 1). Since the velocity of electrons arriving at a target element is determined by the potential of that element, the positive areas are bombarded with high-velocity electrons, and the negative areas with low-velocity electrons, even though electrons originate from the same source.

In order to change the element potential from one level to the other, the "writing" beam must have high current density to overcome the stabilizing 
effects of the holding beam. When the writing beam velocity is high, a positive charge will be deposited on the target; when its velocity is low, a negative charge will be deposited. Thus, the intense writing beam can change the potential of any element (to which it is deflected) either in the positive or in the negative direction, irrespective of the previous condition. Since the erasure is done by the writing beam itself during the writing process, the stored charge pattern can be modified in accordance with the new information without requiring a special erasure process.

In order to reproduce stored information (the process usually referred to as "reading"), the target surface is "probed" with a well-focused electron beam. The net current to the collector, attributable to the "reading" beam, depends upon the transparency of the collector, the number of secondaries generated by the electrons at the target, and the direction and magnitude of the electric field at the two surfaces of the collector electrode. When the reading beam is directed towards a negative area of the target, the secondary electrons from the target pass through the collector screen with a velocity equal to the difference of potential between the negative areas and the collector. In addition, there is a strong suppressing field near the collector. When the reading beam is directed towards positive target areas, the secondaries from the target are not accelerated towards the collector, as is the case near the negative areas, but are in part turned back to the target surface. This occurs under the equilibrium condition since the positive areas of the target are higher in potential than the collector by a few volts because of initial velocities of secondary electrons. The differences in velocities of returning electrons and the differences in electric fields near the collector for the positive and negative areas of the target result in a corresponding difference in the collector current. Thus, the magnitude of the collector current depends upon the polarity of the charge on the target surface, and the probing by the reading beam indicates whether a positive or a negative charge has been stored at the element being probed.

From the above description it can be seen that the Memory Tube can store information in the form of charges on the surface of the target, and the charge distribution can be ascertained by probing with the reading beam. These characteristics make the Memory Tube suitable for use in electronic computers.

The function which the computer memory device is required to perform is to record, to store, and to read out numbers whenever desired, and to record modifications of these numbers which result from mathematical operations upon them. By the use of the binary system any number can be represented by a pattern of 1's and 0's, which can be represented on the Memory Tube target as a pattern of positively and negatively charged "dots." If to each digit is assigned a definite elementary area of the target, then the process of recording a digit consists in deflecting the writing beam to the particular spot and depositing either a positive or a negative charge. The reading process consists in ascertaining with the reading beam the polarity of the charge on the particular element probed.

In order to record and read as many digits as possible, the resolution of the Memory Tube-that is, the number of discrete areas on the target that can be independently charged to either positive or negative potentialshould be as high as possible. This requires that the writing and the reading 
beams be well-focused, and that the target be of such nature that under the action of the holding beam no diffusion of charges over the target areas can take place. In addition, the accuracy in positioning of the writing and reading beams should correspond with the required resolving power. This last requirement makes it very desirable to use the same beam for writing and for reading. This is possible since in the computer tube the writing and reading processes are not simultaneous.

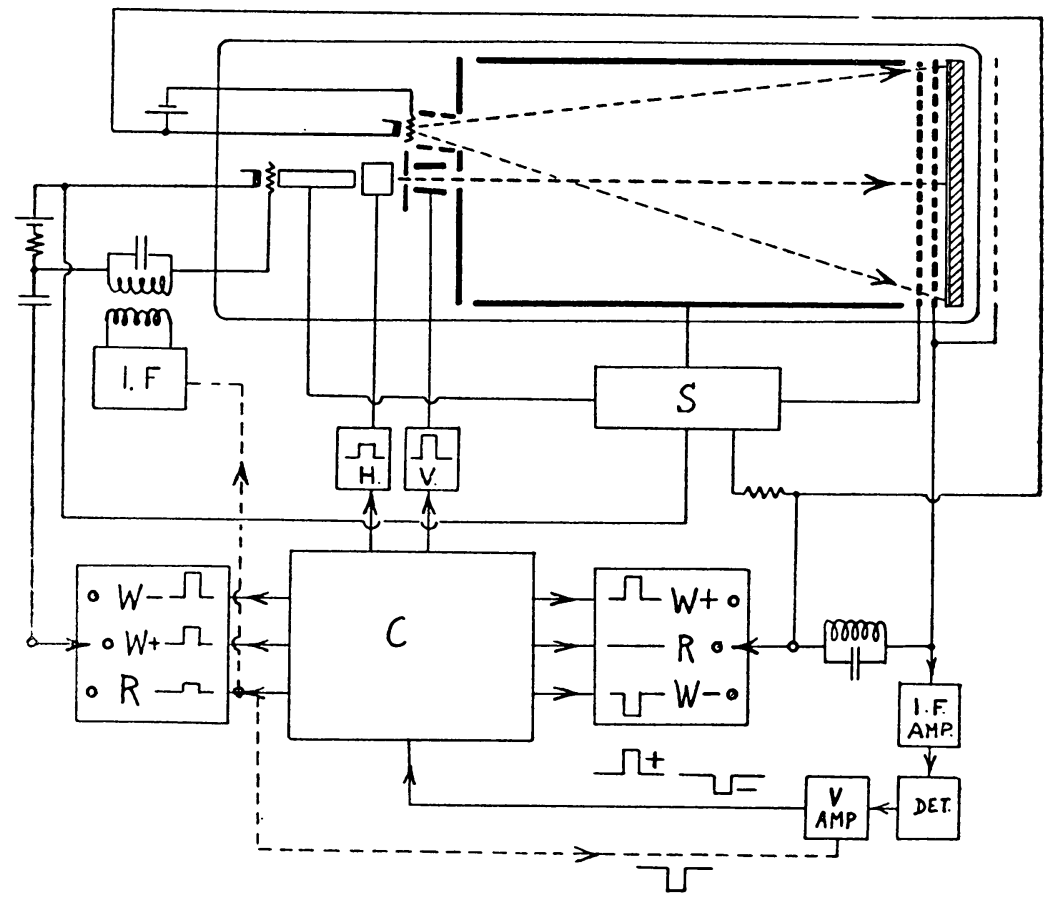

Fig. 2. Schematic Diagram of the Computer Type Memory Tube AND THE Associated Circurts.

A schematic diagram of a Memory Tube intended for computer use is shown in Fig. 2. The write-read gun of the conventional cathode-ray tube type produces a beam focused on a phosphor-coated target. A special holding gun generates a diffuse electron beam, which illuminates the target over its entire area. Two metal woven-mesh screens are placed in front of the target. The screen closest to the target controls the field at the target surface and serves to collect secondary electrons from the target. This screen is ordinarily used as the output electrode. A second screen, farther away from the target, serves to maintain constant the distribution of electric fields along the path of the electron beam irrespective of variable potentials which can be applied to the collector screen. The modulation of collector potential is necessary in order to vary the terminal velocity of electrons for writing either positively or negatively and for reading by means of the same electron beam. The constancy of electric fields along the beam is dictated by the requirement of 
accurate positioning of the write-read beam, irrespective of its terminal velocity.

The diagram of Fig. 2 shows schematically the control pulses originating in the computer represented on the diagram by block $\mathrm{C}$. The different control pulses are necessary in order for the tube to perform the operations of writing, reading, and holding. For writing negatively, a strong positive voltage pulse is applied to the grid of the write-read gun. Simultaneously, a negative voltage pulse is applied to the collector screen. This causes an intense beam of slow electrons to arrive at the target and to deposit a negative charge. In order to deposit a positive charge, the grid of the write-read gun is again pulsed simultaneously with the pulse on the collector, which this time is pulsed in the positive direction. The resulting high terminal velocity of the electrons arriving at the target (which also changes its potential during the pulse because of capacitive coupling between the collector and the target) produces copious secondary emission from the bombarded element of the target and changes it to a positive potential.

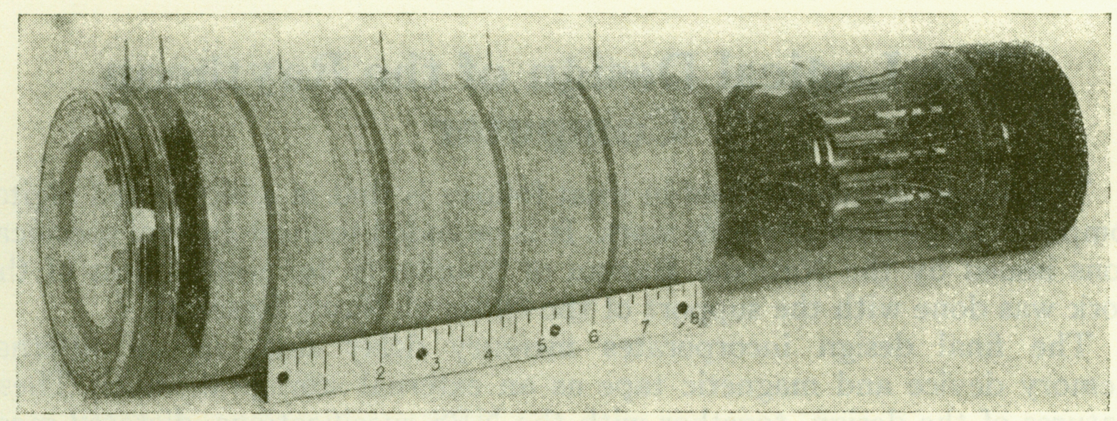

Fig. 3. A Photograph of a Developmental Model of the Memory Tube.

While the terminal velocity of electrons in the writing beam is varied in order to achieve either positive or negative writing, the terminal velocity of electrons in the holding beam should be maintained at a constant value for maximum stability of the charge pattern. This is accomplished by applying to the cathode of the holding gun the same pulse voltages which are applied to the collector electrode, as is shown schematically in Fig. 2.

In order to minimize spurious signals caused by modulating pulses and switching operations during the read-out process, the reading beam is modulated at a frequency of several megacycles. As a result, the output current from the collector screen is also modulated at the same frequency and can be amplified in a selective amplifier (similar to the usual intermediate-frequency amplifiers) which effectively rejects low-frequency components caused by switching. As is shown in the diagram of Fig. 2, a highfrequency voltage is applied to the grid of the write-read gun in addition to a small positive voltage pulse during the read-out process while the collector is maintained at its normal potential. The output signal consisting of an $\mathrm{i}-\mathrm{f}$ modulated voltage pulse appearing across the circuit tuned to $\mathrm{i}-\mathrm{f}$ is amplified in the $\mathrm{i}-\mathrm{f}$ amplifier, then detected and further amplified in a videoamplifier. Since the probing of positive and negative elements of the target 
results in output pulses of different amplitudes, it is possible, by mixing a constant-amplitude negative pulse in the video amplifier, to obtain as a final result a positive pulse for a positive element and a negative pulse for a negative element of the target.

A developmental model of the Memory Tube intended for computer use is shown in Fig. 3. The general shape of the tube, the relative position of the guns and screen-and-target structure can be seen. Since the computer Memory Tube is still in the development stage, it is expected that many improvements in the design of both the tube and the associated circuits will be made before a completely satisfactory performance is achieved. It is hoped that the above brief account of the present state of development will stimulate workers in this field to explore further the potentialities of the memory device which has been made available to them.

Naval Research Laboratory

ANDREW V. HAEFF

Washington, D. C.

${ }^{1}$ A. V. HaefF, “A memory tube," Electronics, Sept. 1947, p. 80-83.

\section{The Logical Design of the Raytheon Computer}

The design to be described in what follows is that of a high-speed digital computer for general scientific work. It is intended to meet general specifications issued by the National Bureau of Standards, for whom most of the work was done with the support of the Office of Naval Research.

The final design incorporates mercury delay lines as an internal memory device and magnetic tape as an external storage medium. These features of the design, together with the NBS specifications, dictated most of the machine parameters.

The basic logical units of the computer are: (1) internal memory, (2) external memory, (3) arithmetic unit, (4) central control, which constitute what might be called the high speed part of the machine, and two auxiliary units: (5) problem preparation unit, (6) printer.

The Memory and the Representation of Numbers and Orders.-The computer handles several different kinds of numbers. In standard or "normal" operation, numbers are stored to a precision of 35 binary digits and with a fixed binary point. The normal precision of the basic arithmetic operation is also 35 digits. Numbers of 70 binary digits-i.e. numbers of double-precision-can be stored and manipulated as pairs of standard numbers. Floating-point numbers can also be handled as pairs of standard numbers, one of these being a number between $\frac{1}{4}$ and $\frac{1}{2}$, and the other being an exponent on 2. Since decimal-to-binary and binary-to-decimal conversions are performed in the arithmetic unit, instead of in auxiliary equipment, decimal numbers must also be stored in the internal memory. These are stored in the binary-coded decimal notation to a normal precision of 8 decimal digits. Decimal numbers of double-precision can be handled as two normal numbers.

Not only numbers but coded instructions (which will henceforth be called orders) as well are stored in the internal memory. An order contains enough information to specify a complete arithmetic operation, including 